\title{
THE PATHOGENESIS OF THE CONTRACTED KIDNEY *
}

\author{
LUDWIG ASCHOFF \\ FREIBLRG, I, BR.
}

In order to obtain a comprehensive insight into the pathogenesis of the various types of contracted kidney in man, it is first of all necessary to attack the problem from the experimental standpoint, and to attempt to produce in animals artificially the identical types of contracted kidney which are observed in human pathology. Unfortunately, up to the present time this has been possible only to an exceedingly limited extent, But in this regard certain pieces of work of the past year or two have served to advance our knowledge, amongst which that of my frienc? Ophüls in San Francisco, of my colleague Mackenzie in Toronto, of their pupil Dickson, and of Christian are especially worthy of mention.

On the other hand, more exact studies concerning the finer morphological structure of the kidney have appeared almost simultaneously in France and in Germany, and these undoubtedly must now form the essential basis for all experimental work. I have also occupied myself with similar studies for several years past, the results of which have in large part been published during the previous year by my pupil Suzuki. My lecture therefore logically resolves itself into three parts:

1. What is our knowledge concerning the morphological structure and the function of the normal kidney?

2. What particular structural elements of the kidney must be damaged to produce chronic contracted kidneys in animals?

3. What is our knowledge concerning the chief types of human contracted kidney?

I shall begin first of all with the question concerning the morphological structure of the renal tubules. As regards the construction of the renal tubular system, we have been accustomed to differentiate the following structural groups: The capsular epithelium, the convoluted tubules of the 1st order (the "Hauptstücke"), the Henle's loop, the convoluted tubules of the $2 d$ order (the "Schaltstücke") and the collecting tubules.

But if we are to take into consideration the newer histological studies and those founded on modern reconstruction technic - and I refer chiefly to those of Policard and Peter - we must elaborate the

* The Cartwright Lectures for the year 1913, of the Association of the Alumni of the College of Physicians and Surgeons, Columbia University.

* Submitted for publication July 21, 1913. 
above classification still further. For Peter divides the ascending limb of Henle's loop into a lower cloudy and an upper transparent portion. $\mathrm{He}$ also calls that narrow tubule which unites the ascending limb of Henle's loop with the Schaltstück, and which usually lies adherent to the glomerular capsule at its hilus, the $Z$ wischenstuick or connecting piece. And, finally, he differentiates the primary collecting tubules from the main collecting tubules, as have some previous authors.

In contrast to this complicated composition of the more distal portions of the tubular system, the Hauptstück (tubulus contortus of the first order) has generally been considered in the literature as an absolute unit; or, at the very most, only the terminal end (the spiral piece of Schweigger-Seidel, partie terminale of Policard) was distinguished from the rest of the Hauptstïck. Even Peter in his schemata of the renal structure in the various animals treats the Hauptstück as a single unit, only giving the terminal portion a special distinction in the cat. Indeed, we find exceedingly scanty information in the literature concerning the finer morphological differences which distinguish this terminal portion.

On the basis of the results obtained from a study of Altmann preparations and vital staining with carmin made in conjunction with Dr. Suzuki, I am now prepared to advise a still further division. For it could be easily determined that in all the animals studied (the rabbit. guinea-pig, rat, mouse, pigeon, to a certain extent also the cat and the hedgehog), the Hauptstiick (convoluted tubule of the first order) can be divided into at least three parts and possibly four.

'The first, or proximal portion, when stained by Altmann's method, is characterized by an extraordinarilly dense and deep staining rod-like structure. On the other hand, the second or middle portion is chararterized by a much more diffuse and more delicate rod-like structure. whereas in the third or distal portion a gradually more and more marked solution into granules takes place, which become progressively more diffuse in distribution. This differentiation into three (or perhaps four) parts stands out still more distinctly in the carmin preparations, so that at first glance one can immediately tell which tubules in the cortex belong to the portions immediately efferent from the glomerulus and which represent the transition to the descending loop. In the rabbit one can still further divide the third or distal portion into a spiral and a straight part.

On the basis of these specific stains, I can only confirm the observation of Peter concerning the more compact arrangement of the tubular convolutions in the deeper glomerular levels, as compared with those lying nearer the surface of the organ. To my mind, the recognition of the characteristic structure of the terminal portion of the Hauptstück is 
particularly important, for, as Peter has also pointed out, the fact that it runs deep into the medulla enables one to confuse it easily with the ascending limb of Henle's loop - a confusion which in certain recent works on vital staining has actually occurred.

Because of the fact that up to the present time the vital staining has been especially used in studying the secretory processes in the kidney, it seemed essential to know exactly where and in what manner the vital staining dyestuffs are cxcreted. From amongst the many dyestuffs that have previously been used, we have purposely chosen carmin, though for the sake of comparison we have also made use of trypanblue. It is, of course, impossible for me to give you the details of each of the exceedingly numerous varieties of experiments performed, nor is it possible at present to describe to you the historical development of this subject or the differences of opinion which still exist on important questions. I desire merely to mention that it was the work of Ribbert and of Arnold which above all others formed for us the starting point for further investigations.

One fact which I desire to emphasize most strongly is that the majority of the authors, though by no means ali, have failed to make a sufficiently sharp distinction between the actual excretion of the dyestuff and the staining of the granules. This distinction is probably one of the most important contributions of our experiments. If the staining of the granules is taken as the index of the dyestuff excretion, then the excretion of carmin may be expected to reach its height twelve to twenty-four hours after the administration of the usual dose employed, for that is the time when a distinct staining of the granules in the cells is first to be observed. On the other hand, since it can be easily determined that only the epithelium of the Hauptstücke shows a granule staining, it seems self-evident that the excretion of the dyestuff takes place through just these cells and through no others. Incidentally, it must here be mentioned, that all observations which would seem to indicate that an excretion of carmin also occurs in the ascending limb of Henle's loop or in the Schaltstücke are based on an error. We should also prefer to believe that similar observations in regard to other dyestuffs such as toluidinblue, trypanblue, etc., are also due to false interpretation of the findings. What has been taken to be the ascending loop is nothing else than the distal portion of the Hauptstück. And in the Schaltstück or in yet more distant divisions of the tubular labyrinth, the dyestuff occurs intracellularly only, when for other reasons we have a right to assume that resorption has taken place.

Indeed, on the results of constantly repeated comparisons of Altmann preparations and carmin preparations from the same kidney, we assert that secretory granule staining occurs only in the epithelium of the Hauptstïck and in the very beginning of the descending loop. The very 
fact that the Hauptstiick stands forth so conspicnously in the vitally stained preparations naturally indicates the possession of a special functional capacity for the excretion of dyestuffs. The variety of the observations in regard to the histological pictures observed during the process of carmin excretion is, in our opinion, to be explained by differences in the doses employed or in the duration of the experiment. If the carmin excretion be systematically followed at intervals of ten minutes, thirty minutes, one hour, etc., for several days or weeks, the following result is obtained. The intensity of the color of the urine and the repeated finding of numerous deeply stained casts of carmin in the collecting tubules of the kidney under observation, definitely indicate that the greater part of the dyestuff is excreted within the first half hour, or at most within the first hour after the injection. In microscopical preparations of kidney obtained after this time, the casts of carmin are either very scarce or entirely absent. Yet in this period when the chief secretion is taking place, no staining of the specific granules is to be seen, or at best only an extremely faint staining. Occasionally, when the dose administered is not too large, no morphological changes whatever are to be seen in the cells, which would indicate a carmin excretion. But if the carmin excretion is very marked, the picture which has been repeatedly described by $\mathrm{A}$. Schmidt, Ribbert and others is to be seen; namely, pink staining of the brushlike marginal membrane (the Bürstensaum), together with an irregular granular precipitate at the base and on the surface of the brushlike margin itself.

The Altmann preparations show that in this period the specific rodlike or granular structure of the cells is unaltered. They demonstrate conclusively that the irregular granular deposit of carmin just described has absolutely nothing to do with the real cellular granules, but represents merely an increased concentration and precipitation within the brushlike marginal membrane which limits the cell interior from the lumen.

The carmin casts which are to be seen in the collecting tubules and occasionally also in the Henle loops present an entirely different appearance, consist of very much finer or coarser granules, and are always more or less uniform in size. The abore mentioned facts all indicate that the dyestuff passes through the epithelial cells of the Hauptstücke in solution, and that only in the lumen of the more distal portions of the tubular system is it thrown down in granular form, due on the one hand to the slowing of the urinary current and on the other to resorptive processes. Here it is usually found embedded in albuminous material. Numerous authors, and more particularly Ribbert, have shown that a resorptive phenomenon probably takes place in the Henle loops, the Schaltstïcke and to some extent also in the collecting tubules. But the final proof that it actually does take place is to be found in the fact that if the 
experiment has lasted some length of time the epithelium of these tubules usually shows the presence of carmin granules, whereas in their lumina casts are still present. The irregularity of their distribution and their coarse appearance render it an easy matter to distinguish them from the secretory granule staining in the epithelium of the Hauptstück.

Although it now seems probable that during the main secretory period the excretion of carmin occurs in soluble form, the question must still be answered whether this excretion occurs exclusively through the epithelium of the Hauptstück, or whether it can also take place elsewhere as well, as, for example, through the glomeruli. As is well known, the occurrence of such a secretion through the glomeruli is at the present time denied. But in the mouse, if the carmin administration be pushed, carmin precipitate can even be encountered in the capsular space. Further, although all the epithelium of the Hauptstücke be severely damaged, as, for example, by chromium, a relatively rapid excretion of carmin will nevertheless still take place. The two facts speak for the assumption that the carmin is excreted in soluble form, not only through the epithelium of the Hauptstiick, but also through the glomeruli, although probably in greater dilution. The fact that this excretion is ordinarily invisible does not speak against it, for if smaller doses are used the excretion can also take place through the epithelium without leaving behind any morphologically demonstrable traces.

We thereby assume a position to a certain extent opposing the presentday teaching, more especially as we desire to emphasize that the period of secretory activity must be sharply distinguished from the period of storing up-the "Speicherungsphase." According to our experience, the phase of secretory activity reaches its height within about one hour and then slowly diminishes, whereas the storing-up phase reaches its height only after twelve to twenty-four hours. From this alone one can conclude that the storing-up process is independent of the secretory process or of the intensity of the secretion. On the contrary, at that time when the cell is being most intensely perfused with the dye fluid, the granules are only very weakly stained, and then gradually the intensity of the staining increases in spite of the progressive diminution of the secretion. Nevertheless, it seems that the storing up of carmin is more or less dependent on a certain optimum rapidity of the intracellular perfusion. Increasing the rapidity of excretion by means of a diuretic immediately results in a prolongation of the period of storing up. Lowering its rapidity, as, for example, by lowering the blood-pressure, results similarly in a delaying of the storing-up process. A faulty staining of the granules by the vital stains may therefore be the result of either of two diametrically opposed functional disturbances, a hyperfunction or a hypofunction. 
We, therefore, arrive at the conclusion that the vital staining of the granules in the renal epithelium has absolutely nothing to do with the actual secretion itself. The prolonged perfusion of the epithelial cells with the dyestuff merely offers the granules the possibility of storing it up. The intencity of the granule staining cannot, therefore, be used as an estimate of functional capacity, as has until now been done, or at best only with very careful reservations. I should like to emphasize that point most emphatically, for even in the most recent work the opinion has been repeatedly expressed that the intensity of the granule staining stands in direct proportional relationship to the functional capacity of the cells. In making such statements the normal physiological differences in intensity with which the dyestuff is stored up in the cell granules of the different divisions of the Hauptstück have, of course, been left entirely out of consideration.

On the other hand, the fact that the storing up of the vital stain occurs in definitely characteristic fashion in each of the different divisions of the "Hauptstück," enables us to solve the important question whether the various renal poisons (cantharidin, arsenic, chromium, uranium, sublimate, etc.) damage the entire "Hauptstück" diffusely, or whether they possess a selective action on certain portions. Here again it is impossible for me to go into details. I desire merely to sum up the matter very briefly by saying that such a selective action does actually occur.

The various renal poisons permit a classification into three groups.

1. Those which damage mainly the terminal portion of the Hauptstück, but whose action may under the influence of larger doses also extend somewhat upward on to the middle division of the Hauptstïck. Very rarely and only when extremely large doses are administered will the action extend on to the proximal division of the Hauptstück as well. To this group belong sublimate and cantharidin.

2. Those in which the damaged area is chiefly limited to the spiral portion of the distal division of the Hauptstücl, spreading downward toward the Henle loops and upward on to the middle division, but rarely reaching as high as the proximal division. Characteristic of this group of poisons is uranium.

3. Those which primarily damage the proximal and middle divisions of the Hauptstiick, though with larger doses spreading downward on the distal and transitional divisions. To this group belongs chiefly chromium.

I should not lay any stress on this specific localization of the action of the various renal poisons, were I not convinced that it possesses an important bearing on the analysis of the function of the kidney. It certainly cannot be an indifferent matter which of the three or four divisions is chiefly damaged by a certain poison, particularly as we know 
absolutely nothing at present concerning what special functions each of these divisions may possess. In fact, on the basis of our numerous series of experiments on the artificial production of hydronephrosis, the injection of uric acid and the observation of physiological forms of epithelial pigmentation (H. Fischer), I believe we have the right to conclude that the various divisions differ from one another in regard to their excretory function.

In this regard $I$ can to-day only refer you to the more detailed publication. I should merely like to mention that uric acid is mainly excreted in the first and second divisions of the Hauptstück and that the lipoid substances, as, for example, in diabetes, are similarly stored up in these divisions. On the other hand, Baehr of New York, when working in my institute, was able to demonstrate that in the diabetic kidney glycogen is not only stored up intracellularly, but also excreted specifically by the cells of the fourth or transitional divisions of the Hauptstück. The work proved that the glycogen deposition in the diabetic kidney is not an incidental degenerative process, situated, as has previously been thought, in the Henle loops. On the contrary, it is an important evidence of a specifically localized secretory process. Of especial interest is the fact, to which Baehr has also called attention, that cantharidin, which acts specifically on this fourth or transitional division, is able to render the kidney relatively impermeable to sugar.

The question concerning the differences in action of the poisons is made still more complicated by the fact that they not only pick out particular portions of the Hauptstïck, but that they also produce different types of damage. For example, chromium, uranium and sublimate produce extensive epithelial necroses in the affected tubules, whereas cantharidin chiefly produces a very marked cellular swelling and vacuolization. And then, finally, even the necroses following the various poisons show differences, in that they may or may not be preceded by a stage of hyalin-droplet degeneration. These facts possess an important bearing on the formation of casts. For in poisonings with those substances which produce the cellular necroses, casts are present in large numbers, whereas in cantharidin and arsenic poisoning they are relatively infrequent. If it were true that cantharidin particularly damages the glomeruli, as most authors have assumed, then the formation of casts following its administration should, according to the teaching of Ribbert, be especially marked. In our numerous experiments with cantharidin we have practically never observed any damage to the glomeruli which was worthy of mention, or which could not similarly be observed following any of the other poisons. We cannot, therefore, agree with those authors who are of the opirion that a glomerulo-nephritis, or a specific damage to the glomerular capillaries of the renal vessels can be produced by the 
use of cantharidin. Our experiments with arsenic have also led to a negative result in this regard.

The actual starting point of our researches along these lines was the work of Schlayer and Hedinger on toxic nephritis several years ago. On the basis of carefully planned experiments, these authors recommended a sharp distinction between vascular and tubular nephritis, which seemed to us to be worthy of a careful histological analysis. We were able to convince ourselves that, on the one hand, the topographical relationship of the damage effected by the different renal poisons employed by these authors was exceedingly varied and complicated; that, on the other hand, the histological picture following cantharidin poisoning showed that with far more justice it deserved to be classed as a tubular poison rather than a vascular, and that finally the great differences in blood-pressure which the authors themselves admit to be present in the tubular and the so-called vascular poisoning is more than sufficient to account for the existing differences in function. For these reasons, it seems to us that the division of the renal poisons into a tubular and a vascular group is not justifiable. We have been forced to the conclusion that all the poisons which we have studied act primarily on the parenchyma, and in fact on particular portions of the Hauptstücke. The variations in the functional disturbance produced by certain of the poisons can be better explained by differences in the topography of the area affected, and by the fact that the individual poisons have a different influence on the blood-pressure.

The knowledge that all the known renal poisons as at present administered act practically exclusively, in so far as is morphologically demonstrable, on the epithelium of the Hauptstücke, destroys all hope of producing experimentally by this means pathological pictures resembling the human nephropathies. Of course, contributions are accumulating in the literature which prove that if administered over a long enough period even these parenchymatous poisons may produce a contracted kidney. But for man it is, on the contrary, just the diseases of the glomeruli which form the most important etiological factor for contracted kidney. Sclerotic areas in the kidney have been produced by Ophüls with lead and with chromium, by Dickson with uranium, by Uyeno under Ponfick's direction with carbolic acid, and the question is still to be settled how these foci of sclerosis are to be explained. One author assumes a direct irritation to the connective tissue, the proliferation of which eventually compresses the epithelial elements. On the other hand, Uyeno and Ponfick believe that the damming up of the tubules by means of casts is the primary cause of the sclerotic areas; that the proliferation occurs in the vicinity of these obstructed tubules, and that this connective tissue increase by gradual contraction and compression causes the tubular shrinkage. 
The pathological pictures which we obtained in chronic uranium poisoning thoroughly convinced us that neither of these two hypotheses represent the real causative factor, although it cannot be denied that the retention of casts is of some significance. The great majority of the casts, however, are flushed out of the organ in a relatively short time and only occasionally may some remain behind, become calcified and thereby incite a reactive inflammation in its vicinity which may lead to total obliteration of the lumen. We feel convinced that still another process plays the chief rôle. In accordance with the work of the most recent authors, amongst which that of Thorel, of Heinecke and of Tilp must be especially mentioned, we were able to observe the extraordinarily rapid epithelial regeneration in the damaged Hauptstücke in all animals. and could readily determine that this epithelialization had its point of origin primarily in the narrow limb of Henle's loop and in the remains of the lower transitional division of the Hauptstiuck. Should the advance of the proliferating epithelium be obstructed by impregnable necrotic masses, or the epithelium from which the regeneration ordinarily starts be too severely damaged, there then results a collapse, and, eventually, total destruction of that particular entire tubular system. The portion of the tubular system proximal to the point of obstruction undergoes a pressure and inactivity atrophy absolutely identical with that occurring in hydronephrosis. And in this manner the numerous atrophic islands in the renal cortex develop, which are particularly characterized by the fact that the glomerulus belonging to the atrophic focus is somewhat smaller than normal, but otherwise strikingly well preserved, that its capsule is thickened, and its capsular space dilated. Although the process I have just described leads to a tubular contracted kidney as distinguished from a glomerular, it nevertheless does in my opinion find its analogy in human pathology in the pyelonephritic contracted kidney, the uric acid kidney and the sclerotic foci proximal to renal cysts, ete.

But since the glomerular contracted kidney is, as a matter of fact, really the predominating type in human pathology, the most important problem was to find a means of producing a glomerulonephritis in animals. I have already mentioned that the glomerular lesions observed after the administration of the ordinary renal poisons can by no means be considered a genuine glomerulonephritis. Only in uranium nitrate poisoning have Christian and Mackenzie been able to observe certain changes in the glomerular loops which would seem to indicate a possible vulnerability of the glomeruli in regard to uranium.

Baehr has recently been studying these various problems in the Freiburg Institute in greater detail. By the subcutaneous, but above all by the intra-arterial administration of uranium, he has succeeded in producing experimentally absolutely typical pictures of human glomerulo- 
nephritis, and especially of the Löhlein type of glomerular lesion occurring with chronic ulcerative thromboendocarditis. The process begins with blood platelet thrombi in the capillary loops, necroses of the endothelial capillary walls and exudation into the capsular space. Then follows absolutely characteristic proliferation of the epithelium of Bowman's capsule with the formation of typical epithelial crescents. And, finally, there result adhesions which lead to partial obliteration of the capsular space. Hemorrhages also accompany the glomerular changes, so that even macroscopically the characteristic red punctate dotting of the cortex is to be observed. Unfortunately thus far the animals have usually died within the first few weeks, so that no very faradranced stage of contracted kidney has as yet been obtained. But Baehr has for the first time produced glomerulonephritis experimentally, and his researches are the first which point the way to the experimental production of glomerular contracted kidneys. Of particular interest is the fact that by similar intra-arterial injection of iodin, chromium and croton oil no such glomerular changes could be produced, so that uranium must possess a specific action.

Damage to the glomerular apparatus must by no means be considered as identical with damage to the renal vessels, as some authors have wrongly assumed, and there have also been attempts to injure only the vessels themselves. As I have already mentioned, it was especially Schlayer who believed that in cantharidin and arsenic he possessed poisons which specifically damage the renal vessels. But as Pearce, Hill and Eisenbrey have shown, an absolutely sharp distinction between vascular and tubular poisons cannot be made. Furthermore, a critical study of Schlayer's experiments, which Baehr has recently undertaken, has also shown that among other things the general circulatory disturbance produced by the so-called vascular poisons is one of the chief causes of the early oliguria and other functions! changes on which this classification is based. We possess at present no means with which to damage the renal vessels alone, and, therefore, no means with which to induce in animals a reproduction of the vascular type of contracted kidney.

It must, therefore, be frankly recognized that up to the present time only tubular contracted kidneys have been experimentally produced in animals, but that on the basis of Baehr's experiments we can also expect. to produce glomerular contracted kidneys in the near future. Only then will it be possible to study satisfactorily the effect of such renal changes on the general organism, and, what will interest the clinician most of all, their influence on the vascular system, on the heart, and on the adrenals. 
For the time being we are, therefore, obliged to confine ourselves to the pathological findings in our human material. But even in human pathology there exists up to the present time such a confusion in terminology and in interpretation that a satisfactory understanding of kidney diseases is a very difficult matter. This is the reason for the oft-repeated complaint that we possess no clear and reliable classification of renal diseases, and that we make no sharp distinction between the actual inflammatory processes and the other pathological disturbances which affect the kidney. It was no less a man than Friedrich Müller who, at the Meran Congress of the "Deutschen Naturforscher und Aerzte," distinctly advocated a division of the non-inflammatory from the inflammatory kidney affections under the names of the "Nephroses" and the "Nephritides," respectively. But the term nephrosis has not come into general use, and in order to preserve a terminology analogous to that for diseases of other organs, it would be better to replace it by the term "Nephropathy."

Inasmuch as we know so very little concerning the etiological factors, it is permissible to classify the nephropathies on the basis of pathogenesis. We can best divide all diseased conditions of the kidney into those which are purely or mainly passive in nature, and those which are reactive or inflammatory in nature. The first group can be again divided into the disturbances in development and form, the disturbances in circulation and the metabolic disturbances; the second group, which is really a complicated mixture of passive and active processes, must also be arranged into subdivisions according to whether the inflammatory reaction chiefly limits itself to the blood-vessels and connective tissue, or to the glomerular apparatus or to the tubular apparatus with its own individual divisions.

Finally, according to the course of the pathological process, some of the subdivisions must be separated into the acute and the chronic affections. Although it is extremely tempting to compare for you the acute renal diseases with those facts which have been determined experimentally in animals, I nevertheless feel that I must confine myself to a brief sketch of the chronic renal affections, because, in default of material for satisfactory comparison in the field of experimental work, such a brief outline seems more necessary for a better understanding of the present-day conceptions. I have attempted to present a survey of the chronic renal affections alone, at the same time indicating the acute processes from which these develop. Although in the chronic affections of mainly passive origin - those of formative, degenerative or circulatory origin - the pathological condition under consideration usually develops

1. As used by me in 1909, and recently recommended by Barker (Am. Jour. Med. Sc., 1913, cxlv, 42 ). 
insidiously from the very onset; the chronic affections of inflammatory origin are, on the contrary, usually ushered in by an acute process. The various forms of inflammatory contracted kidney result secondarily therefrom, and ought really to be considered as nothing more or less than cicatrices in various stages of development. In fact, the inflammatory contracted kidneys resemble the other chronic nephropathies of passive nature in this respect, for the reactive process is more or less completely extinguished and only a physical disproportion and perhaps a certain disturbance in functional equilibrium remains.

It is not my purpose to enter on a detailed description of these various chronic renal affections, referring you rather to the text-books of pathological anatomy in which the frequent and confused misunderstandings, as, for example, that in regard to chronic parenchymatous nephritis, have been subjected to a searching exposition in the light of recent investigations. But I desire to call your attention to one point, namely, the great variety which the several forms of contracted kidney present in regard to their pathogenesis, although they form only one subdivision of the ehronic renal affections. I shall limit myself to a comparison of the genuine and the secondary contracted kidney.

\section{THE CHRONIC NEPHROPATHIES}

I. Nephropathies of formative origin (disturbances in development and form).

Examples: 1. Nephropathia cystica. Congenital cystic kidneys.

2. Nephropathia hydronephrotica. Hydronephrotic contracted kidneys.

3. Nephropathia carcinomatosa, ete. Carcinoma of the kidney, etc.

II. Nephropathies of degenerative origin (metabolic disturbances).

Examples: 1. Nephropathia diabetica. Glycogen excretion and deposition in the lowermost division of the "Haupstück" (convoluted tubule of first order).

2. Nephropathia urica. Uric acid collections in the resorbing portions of the renal tukules.

3. Nephropathia amyloidea. Amyloid degeneration of the capillary system.

4. Nephropathia Basedowiana. Fat infiltrations in the secretory portions of the renal tubules.

III. Nephropathies of vascular origin (circulatory disturbances).

Examples: 1. Nephropathia albuminurica orthostatica adolescentium.

2. Nephrosclerosis cyanotica. Kidney of passive congestion.

3. Nephrocirrhosis embolica. Embolic contracted kidney.

4. Nephrocirrhosis arteriosclerotica. Arteriosclerotic contracted kidney.

5. Nephrocirrhosis genuina. Genuine or primary contracted kidney. Granular atrophy. Presenile sclerosis affecting the smallest branches of the renal artery. 
IV. Nephropathies of inflammatory origin.

Examples: 1. Nephrocirrhosis glomerularis. Glomerular or secondary contracted kidney. (The "chronic parenchymatous kidney" of older authors). Develops after an acute glomerulonephritis.

2. Nephrocirrhosis tubularis. Tubular contracted kidney. Develops after an acute tubular nephritis. Exceedingly rare in man, if it occurs at all. In animals it can be experimentally produced by chronic uranium poisoning, and by intraarterial injection of iodin.

3. Nephrocirrhosis apostematica. Pyelonephritic contracted kidney. Develops after an acute suppurative inflammation of either hematogenous or urinogenic origin.

4. Nephrocirrhosis tuberculosa, etc. Tuberculous contracted kidneys, syphilitic, ete.

Even at the present day the idea is still widespread that the genuine or primary contracted kidney is the result of an inflammatory process, and the possibility cannot be denied that such a mode of origin may occasionally occur, as, for example, following the acute exudative lymphocytic or leukocytic nephritides of scarlet fever and of streptococcus infection, a possibility which even I myself have mentioned in my textbook. But it is now, as a matter of fact, beyond the shadow of a doubt that the majority of the uncomplicated cases develop as a result of a vascular disturbance, or, to be more exact, from a sclerosis of the finest branches of the renal artery, beginning relatively early in life and leading secondarily to the formation of atrophic foci in the parenchyma and to hyaline degeneration of the glomeruli, with a resultant focal inactivity atrophy of the tubules belonging to them. These facts, originally brought forward by Jores, have been thoroughly confirmed by Loehlein, Herxheimer, Fahr and Gaskell. This is, therefore, no primary disease of the filter apparatus; that is, of the glomeruli, but of the blood-vessels leading to the filter, a presenile arteriosclerosis of the finest branches of the renal artery.

The old teaching of Gull and Sutton concerning the arteriocapillary fibrosis in the "red contracted kidney" is herewith again brought to the front and properly honored. But, indeed, what induces primary disease of the smallest arterioles is still an unsolved problem. Is it only one manifestation of a general disease of all the smallest arteries (of the pancreas, retina, brain, etc.)? Or does it precede all the others and only secondarily, by reducing the volume of the afferent flow of blood to the renal filter, either reflexly or chemically call forth a constant high bloodpressure with its consequences, the classical cardiac hypertrophy and the sclerosis of the peripheral arterioles due to overwork and overuse? And if it does precede, to what is it then due? Is it the result of a congenital inferiority of the renal vascular apparatus, or is perhaps the filter relatively too small and its total diameter too narrow for the volume of its afferent blood-current? Is it perhaps due to some specific effect of 
the ingested food or of endogenous irritants on the afferent blood-vessels of the renal filter? These are the problems which are still waiting for solution, as is also the question concerning the relation of the entire process to the chromaffin system. Only one thing seems certain, and that is that the renal sclerosis and contraction is primarily passive in nature, the result of the disease of the renal ressels. In this respect, this type of contract kidney approaches the arteriosclerotic, in which the disease, as one might say, spreads from the aorta outward into the individual branches of the renal artery and so only induces an inactivity atrophy of isolated, but larger areas of the kidney parenchyma.

Nevertheless, the amount of the filter which is incapacitated because of the peripheral disease of the renal vascular system in the genuine contracted kidney is far greater than that in the arteriosclerotic, and for this reason the latter does not induce such an outspoken secondary effect on the general vascular system and the heart. The larger the amount of filter, that is, of glomerular tissue functionally incapacitated, the greater must be the circulation through the remaining filter tissue in regard to blood-pressure and speed of the current, in order to keep up the amount of excretion necessary for the organism. This remaining filter tissue works on the borderline of its maximum functional capacity as long as its blood-supply is maintained at the proper speed and under a sufficiently high pressure. Hence, the normal output of urinary fluir and even polyuria. But it must be borne in mind, that although the kidney performs an increased work output, similar to that in cardiac hypertrophy, this always remains within definite limits and can, indeed, be chiefly increased by influencing the heart. This accounts for the relatively limited power of accommodation in the polyuric vascular contracted kidneys. Even the tubules that remain undamaged work more or less at their maximum. This interpretation of the process at least permits us to explain the frequent, and often very marked compensatory hypertrophy of the remaining glomeruli, as well as the remaining tubules, in such contracted kidneys.

The problem of the secondary contracted kidney is an entirely different matter; the primary factor is here an inflammatory change affecting the renal filter apparatus itself. The significance of this initial glomerular disease, attention to which had previously been called by Nauwerk, v. Kahlden, Ribbert, Reichel, etc., has recently again been particularly emphasized by Loehlein; and the subsequent studies of Gaskell, Baehr and others, have supplied ample confirmation of its importance. But these inflammatory contracted kidneys do not alone differ from the genuine vascular contracted kidneys in that the primary seat of the disease is in the glomeruli. One of their chief characteristics is usually the general involvement of the entire filter apparatus by the 
pathological change, so that the reparative and the regenerative compensatory processes find much more difficulty in effecting a restoration of the normal function. For this reason the slightest overexertion, or even the physiological variations in renal work may very easily cause the renal tissue to quit work, thereby inducing uremia and the characteristic edema. The clinical picture is usually dominated by a glomerular oliguria, although if a sufficiently large part of the filter has recovered, this may give way to a polyuria. And this polyuria, exactly like that accompanying the genuine contracted kidney, depends on overwork of the remaining normal portions of the filter apparatus under the influence

Table Showing Localization of the Action of Various Toxic Agents on the Uriniferous Tubui.es of the Rabbit's Kidney

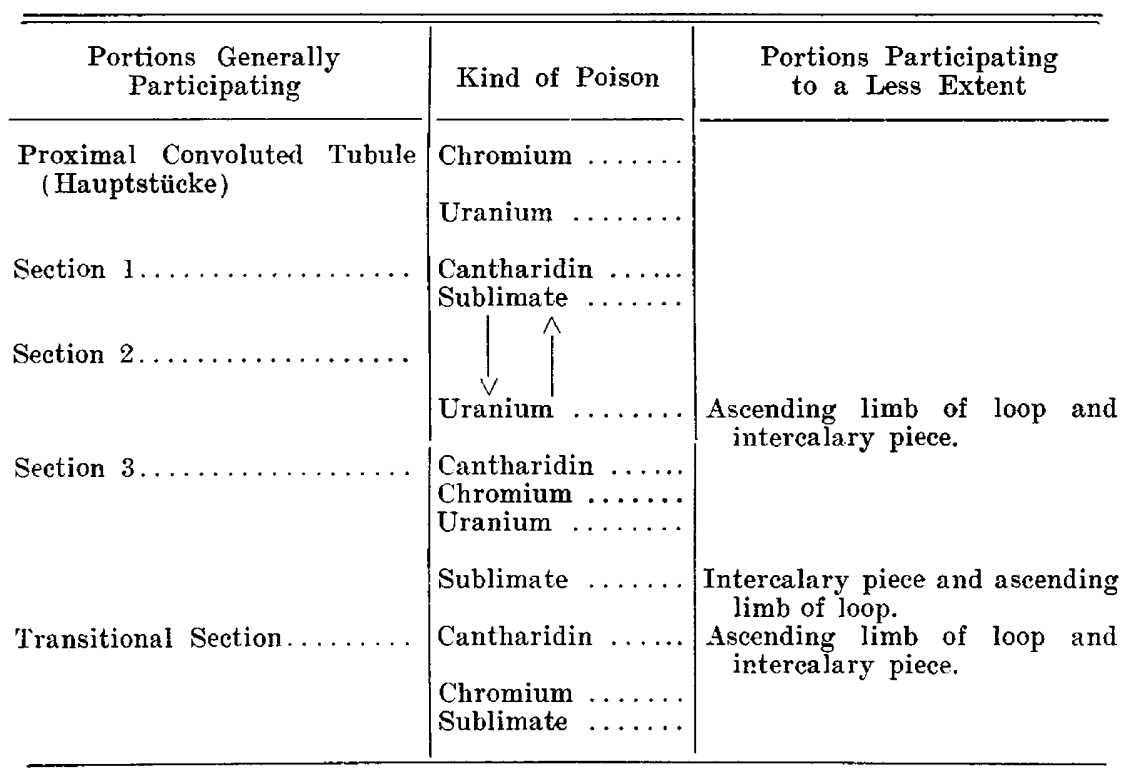

of an increased circulation through them, combined with an increased blood-pressure in the entire general vascular system. All our functional tests will actually test oniy these remaining normal portions of the filter apparatus which are working more or less at their maximum, and their particular vessels; but it must be remembered that they do not test the diseased tissue which is now in the scar stage.

Here in the secondary contracted kidney, the tubular secretory tissue connected with each diseased glomerulus is similarly put out of function, just as in the genuine contracted kidney, but usually to an even far more widespread extent.

The above considerations indicate that in the severe forms of nephritis oliguria depends on a glomerular disease and not on a disease of the 
blood-vessels. Also the polyuria, both in the nephritic as well as in the vascular contracted kidney, in so far as the histological findings seem to indicate, does not depend on a hypersensitiveness of the still diseased vessels, but on the increased work of the undamaged vessels and their respective filter apparatus, or the only slightly damaged ones, or those which have entirely recovered from their damage.

These forms of polyuria, which I might term compensatory or accommodative in nature, must be sharply distinguished from the acute irritative polyurias of toxic origin. The latter depend on a local damage to the blood-vessel filter apparatus, the former on a functional accommodation, usually accompanied by simultaneous accommodative processes in the rest of the general vascular system.

I hope that with these few examples I have made it clear that the problem of acute nephritis is entirely different from that of the chronic nephropathies, just as in toxicology the problem of acute poisonings differs from that of the chronic. In the acute nephritides we have to do with the question of an acute damage to previously normal functions; in the chronic nephropathies the question is that of the possibilities for compensation and of a failure of these compensatory regulations. The opportunity to penetrate more deeply into these two problems and to bring forth the solution has now been made possible by the recent advances in our knowledge concerning the normal structure of the organ and the experimental production of tubular and glomerular contracted kidneys. 